\title{
Charge Regulation at a Nanoporous Two-Dimensional Interface
}

\author{
Mandakranta Ghosh, Moritz A. Junker, Robert T. M. van Lent, Lukas Madauß, Marika Schleberger, \\ Henning Lebius, Abdenacer Benyagoub, Jeffery A. Wood,* and Rob G. H. Lammertink*
}

Cite This: ACS Omega 2021, 6, 2487-2493

Read Online

ABSTRACT: In this work, we have studied the pH-dependent surface charge nature of nanoporous graphene. This has been investigated by membrane potential and by streaming current measurements, both with varying $\mathrm{pH}$. We observed a lowering of the membrane potential with decreasing $\mathrm{pH}$ for a fixed concentration gradient of potassium chloride $(\mathrm{KCl})$ in the Donnan dominated regime. Interestingly, the potential reverses its sign close to $\mathrm{pH} 4$. The fitted value of effective fixed ion concentration $\left(\bar{C}_{R}\right)$ in the membrane also follows the same trend. The streaming current measurements show a similar trend with sign reversal around $\mathrm{pH}$ 4.2. The zeta

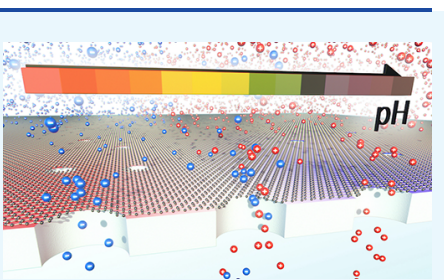
potential data from the streaming current measurement is further analyzed using a 1-pK model. The model is used to determine a representative $\mathrm{pK}$ (acid-base equilibrium constant) of 4.2 for the surface of these perforated graphene membranes. In addition, we have also theoretically investigated the effect of the PET support in our membrane potential measurement using numerical simulations. Our results indicate that the concentration drop inside the PET support can be a major contributor (up to $85 \%$ ) for a significant deviation of the membrane potential from the ideal Nernst potential.

\section{INTRODUCTION}

Perforated monolayer graphene is a two dimensional material in which pores have been created in a controlled manner by, e.g., heavy ion beam bombardment, focused ion beams, electrical pulse method, and oxygen plasma etching. ${ }^{1-7}$ Nanoporous graphene membranes have potential applications in the fields of separation, filtration, and biomolecular translocation..$^{8-11}$ As ions can diffuse through these pores in graphene, it can be used as electrodes for lithium ion batteries, spacers, as well as supercapacitors. ${ }^{12,13}$ To achieve all these potential applications in practice, it is important to study the transport characteristics through these two-dimensional nanoporous materials. In our previous work, we have investigated the ion transport properties of perforated graphene by varying the concentration of monovalent and bivalent cations to understand how the single-layer membranes behave. ${ }^{14,15}$ For all the salts under investigation $\left(\mathrm{KCl}, \mathrm{LiCl}, \mathrm{K}_{2} \mathrm{SO}_{4}, \mathrm{MgCl}_{2}\right.$, $\mathrm{CaCl}_{2}$, and $\mathrm{NH}_{4} \mathrm{Cl}$ ), we observed clear Donnan and diffusion dominated regimes due to Donnan exclusion of ions and differences in the self-diffusion coefficients of ions, respectively. These membranes further exhibited strong adsorption phenomena for bivalent cations. A further measurement of the bi-ionic potential indicated that there are differences in the interaction of ions with the graphene surface, which lead to the differences in the Donnan-dominated plateaus. We could also quantify the extent of differences in the selectivities of different ions and relate it to the ratio of their ideality factors. This ideality factor $(\alpha)$ is an empirical correction factor related to the deviation of the measured membrane potential vs the expected value in the Donnan plateau, i.e., the Nernst potential. ${ }^{14}$ Through the use of numerical simulations based on the Poisson-Nernst-Planck equations, we demonstrate that $\alpha$ arises from a combination of the typical magnitude of surface potentials and from concentration gradients within the PET support used.

All these experiments conducted so far, were done at $\mathrm{pH}$ neutral conditions, where the membrane was found to be cation selective. The cation selective nature implies the presence of fixed negative charges at the surface. These charges are possibly introduced during the fabrication process (e.g., deprotonation of surface hydroxyl or carboxyl) or by adsorption of anions on the membrane surface. This motivates us to further investigate the nature of surface charge present at these nanoporous graphene membranes and its effect on ion transport. The charge of these surface groups is expected to be subjected to acid-base equilibrium. The influence of $\mathrm{pH}$ can be related to the surface $\mathrm{pK}$, which represents the surface acid dissociation constant. So far, there are very few studies on the surface charge groups present on the nanoporous monolayer graphene as the conventional experimental techniques, including titration or FTIR techniques, are not suitable for single-layer graphene. Most studies to date have concerned graphene oxide (GO), reduced graphene oxide ( $\mathrm{GGO}$ ) or are purely based on MD simulations. ${ }^{16-18}$ Konkena et al. have shown that GO sheets contain acidic groups with a $\mathrm{pK}$ of 4.3 and groups with $\mathrm{pK}$ values of 6.6 and 9.0 by conducting zeta potential measurements, $\mathrm{pH}$ titration, and infrared spectros-

Received: August 17, 2020

Accepted: December 31, 2020

Published: January 20, 2021 
copy. ${ }^{16}$ Shih et al. have investigated the surface activity with varying $\mathrm{pH}$ through $\mathrm{MD}$ simulations. ${ }^{17}$ Their result shows that, at low $\mathrm{pH}$, the carboxyl groups become less hydrophilic and form aggregates. Orth et al. determined the $\mathrm{pK}$ of graphenelike materials via titration and the obtained $\mathrm{pK}$ values match with carboxylic acid groups, less acidic carboxylic groups, and alcohol groups. ${ }^{18}$

It is well known for pristine graphene to be highly hydrophobic due to the absence of functional groups, making processing in water difficult. Therefore, graphene is typically functionalized using covalently bound groups (e.g., graphene oxide) or non-covalently bound groups (e.g., surfactants) to enable dispersion stability in water. ${ }^{19-21}$ Bepete et al. were able to produce stable dispersion of single-layer graphene in water without functionalization via electrostatic stabilization. ${ }^{22}$ Here, the surface charging mechanism was proposed to originate from hydroxide ion adsorption. Charge reversal was observed around $\mathrm{pH} 4$, which is typical for inert hydrophobic surfaces in an aqueous environment, caused by competitive adsorption of hydroxide and hydronium ions. ${ }^{23}$ Rollings et al. have shown that for a $3 \mathrm{~nm}$ pore in graphene fabricated by an electrical pulse method, the $\mathrm{K}^{+} / \mathrm{Cl}^{-}$selectivity shows a sharp decrease

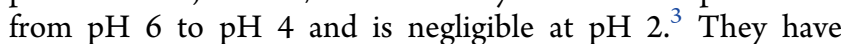
attributed this effect to protonation of a surface charge group (e.g., carboxyl) present at the graphene edge, leading to an effective reduction in the charge density of their pores.

Here, we present the measurement of membrane potential induced by salt $(\mathrm{KCl})$ concentration gradients across perforated graphene membranes at different $\mathrm{pH}$ values. These measurements provide direct insight on the surface charge state of the graphene membrane. We have corroborated our experimental results with streaming current measurements at a fixed salt concentration and varying $\mathrm{pH}$. The extracted zeta potentials show a similar influence of $\mathrm{pH}$ compared to the membrane potential. From the zeta potential data, a surface $\mathrm{pK}$ value was fitted for our graphene surface. ${ }^{24-26}$ In addition, we performed numerical simulations to evaluate the effect of diffusion resistance of the PET support on the observed membrane potential. We show that, for a plausible range of surface potentials of a graphene nanopore, this diffusion resistance can be a major contributor to the deviation of ideal vs measured potential. Our simulations also demonstrate that diffusion within the PET pore cannot explain the observed charge inversion in graphene at different $\mathrm{pH}$ values.

\section{THEORY}

Two frequently used charge regulation models for surfaces in contact with electrolyte solutions are the $1-\mathrm{pK}$ and 2 -pK models. $^{26}$ The major difference between these models is the considered changes in the protonation states of a representative functional group. As implied by the name, the 1-pK model considers one protonation step, with the charge varying between $-1 / 2 \mathrm{e}$ and $+1 / 2 \mathrm{e}$. The 2 -pK model accordingly accounts for two protonation steps, with the charge varying between $-1 \mathrm{e}, 0$, and $+1 \mathrm{e}$. Hence, at the cost of one additional fitting parameter, the $2-\mathrm{pK}$ model, at least in theory, allows for a more accurate representation of the experimental data. However, it was shown by Piasecki and Rudzinski ${ }^{26}$ that even, under consideration of various experimental methods, both models can work equally well, although resulting in somewhat different physical parameters. Therefore, the physical correctness of the model and the resulting parameters should be taken with care. In light of the limited characterization methods conducted in this work, it is reasonable to limit the number of fitting parameters to ensure a unique solution. Thus, the 1-pK model is used in combination with the basic Stern model (BSM), which describes the electric double layer forming at the graphene/electrolyte interface. Neglecting other ion adsorption, this results in the following set of equations: ${ }^{26}$

$$
\begin{aligned}
& { }^{1} K_{H}^{\text {int }}=\frac{\left[S O H^{(1 / 2)-}\right] \cdot a_{H}}{\left[S O H_{2}^{(1 / 2)+}\right]} \cdot \exp \left(\frac{-e \Phi_{0}}{k_{\mathrm{B}} T}\right) \\
& \Phi_{0}-\Phi_{d}=\frac{\sigma_{0}}{C_{1}} \\
& \Phi_{d}=\frac{2 k_{\mathrm{B}} T}{|z| e} \cdot \ln \left(\frac{-\sigma_{d}}{\sqrt{8 \varepsilon_{0} \varepsilon_{r} k_{\mathrm{B}} T I}}+\sqrt{\frac{\sigma_{d}^{2}}{8 \varepsilon_{0} \varepsilon_{r} k_{\mathrm{B}} T I}+1}\right) \\
& \sigma_{0}=\frac{1}{2}\left(e \cdot\left[S O H_{2}^{(1 / 2)+}\right]-e \cdot\left[S O H^{(1 / 2)-}\right]\right) \\
& \sigma_{d}=-\sigma_{0} \\
& N_{s}=\left[S O H_{2}^{(1 / 2)+}\right]+\left[S O H^{(1 / 2)-}\right]
\end{aligned}
$$

Here, $\mathrm{SOH}$ refers to an amphoteric surface hydroxyl group, which can associate and dissociate a proton. $\left[\mathrm{SOH}^{(1 / 2)-}\right]$ and $\left[\mathrm{SOH}_{2}^{(1 / 2)+}\right]$ are the number of negative and positive functional groups on the surface $\left(\mathrm{m}^{-2}\right)$, where $\mathrm{SOH}_{2}^{(1 / 2)+}$ refers to the surface site formed by the protonation reaction. ${ }^{1} K_{H}^{\text {int }}$ is the equilibrium constant of protonation, which is determined by the point of zero charge (PZC), $a_{H}$ is the proton activity in the bulk solution $\left(\mathrm{mol} / \mathrm{dm}^{3}\right), \Phi_{0}$ and $\Phi_{d}$ are the electrical potentials at the surface and outer Helmholtz plane, $\sigma_{0}$ and $\sigma_{d}$ are the charge densities $\left(\mathrm{C} / \mathrm{m}^{2}\right)$ at the surface and inside the diffusive boundary layer. The two fitting parameters are the Helmholtz capacity $C_{1}$ and the total density of functional groups $N_{s}$. All other physical constants have their typical denotation with $\varepsilon_{r}$ being 78 (relative permittivity of water at 25 $\left.{ }^{\circ} \mathrm{C}\right), \varepsilon_{0}$ the vacuum permittivity, and $I$ the ionic strength of the solution $\left(\mathrm{m}^{-3}\right)$. Assuming $\zeta \approx \Phi_{d}$, the system of equations is iteratively solved and fitted to the experimental zeta potential data via a least-square approach using a gradient-free search algorithm.

\section{EXPERIMENTAL PROCEDURE}

For our study, we have used single-layer graphene supported on a PET substrate. These membranes were irradiated with 5 $\times 10^{8}$ ions $/ \mathrm{cm}^{2}$ using ${ }^{129} \mathrm{Xe}^{23+}$ ions having a specific energy of $0.71 \mathrm{MeV} / \mathrm{u}$. This irradiation creates pores in the nanometer range in the graphene. After etching, the PET pores are around 20-40 times larger than the graphene pores. The PET pore can have an influence on the resulting ion transport, as will be discussed later as well as in the Supporting Information. For further details of the fabrication process, please look at our previously published papers. ${ }^{2,14}$

3.1. Membrane Potential with Varying $\mathrm{pH}$. The membrane is placed between two reservoirs containing electrolyte solutions of different concentrations. The high and low $\mathrm{KCl}$ concentration solutions are circulated in each compartment and through a $25{ }^{\circ} \mathrm{C}$ temperature bath. The high and low electrolyte concentration compartments are kept at a ratio of 5:1. Calomel reference electrodes are used to measure the potential across the membrane using a potentiostat (Autolab PGSTAT302N). The $\mathrm{pH}$ is adjusted using $\mathrm{HCl}$ 
and $\mathrm{NaOH}$ solutions. The $\mathrm{pH}$ and conductivity of the solutions are measured before and after the membrane potential measurements to make sure that these two remain constant throughout the measurement.

3.2. Estimating Zeta Potential vs $\mathbf{p H}$. Streaming current measurements are carried out by a SurPASS electrokinetic analyzer (Anton Paar). ${ }^{27,28}$ The setup contained an adaptable gap cell with two sample holders where two graphene membranes are placed facing each other. The flow of electrolyte solution is adjusted over the cell by two syringe pumps. In order to measure the streaming current with varying $\mathrm{pH}, \mathrm{HCl}$ and $\mathrm{NaOH}$ solutions are added to the $\mathrm{KCl}$ solution in an automatic fashion by the device software. The zeta potential of the surface was estimated from the streaming current vs pressure data based on eq $7:{ }^{29}$

$$
\zeta=\frac{\mathrm{d} J}{\mathrm{~d} \Delta p} \frac{\eta}{\varepsilon_{0} \varepsilon_{r}} \frac{L}{A}
$$

where $\frac{\mathrm{d} J}{\mathrm{~d} \Delta p}$ is the slope of streaming current vs differential pressure, $\eta$ is electrolyte viscosity, $L$ is the length of the streaming channel, and $A$ is the cross-sectional area.

\section{RESULTS AND DISCUSSION}

Figure 1 shows the variation of the normalized membrane potential $\left(\Delta \Psi / \Delta \Psi_{\text {Nernst }}\right)$ with $\mathrm{pH}$ at different electrolyte

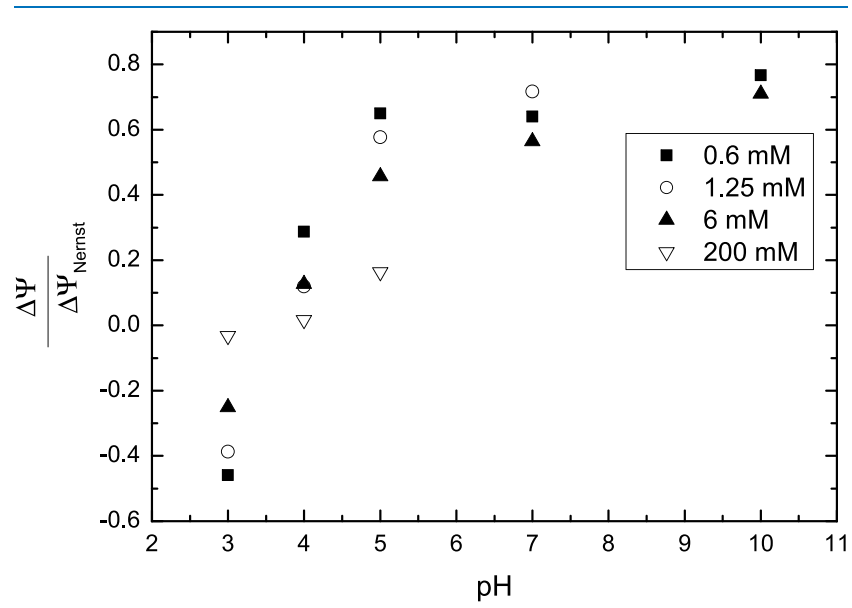

Figure 1. Scaled membrane potential with Nernst potential with varied $\mathrm{pH}$ at different $\mathrm{KCl}$ electrolyte concentrations at the constant ratio of 5 . The concentrations in the legend are concentrations of the low concentration side of the reservoirs.

concentrations (at a fixed ratio of 5). The Nernst potential refers to the theoretical potential one would obtain for a perfectly selective membrane exposed to an ion concentration ratio of 5 to 1 . We stress that the actual concentration ratio that will be located across the graphene pore can be affected by an ion concentration gradient inside the PET support pore. Such a gradient in the PET pore will reduce the gradient across the graphene pore and hence reduce the measured potential. This effect and the possible non-perfect selectivity of the graphene can cause the observed scaled membrane potential below unity. A further analysis of the pore geometry and its potential influence on the membrane potential is provided in our current study. Additional $\mathrm{HCl}$ and $\mathrm{NaOH}$ concentrations in both reservoirs are taken into account to calculate the Nernst potential $\left(\Delta \Psi_{\text {Nernst }}\right)$ as these can affect the ion concentration ratio between reservoirs. As the concentrations of protons and hydroxide are equal for both reservoirs, there is no bulk concentration gradient for these ions but there can be a local one in the PET pore. The formula to calculate $\Delta \Psi_{\text {Nernst }}$ is given in the Supporting Information as well as their values for different concentrations and $\mathrm{pH}$ values.

Figure 1 shows that the scaled membrane potential is almost constant in the $\mathrm{pH} \mathrm{5-10} \mathrm{range} \mathrm{for} \mathrm{low} \mathrm{KCl}$ concentration range $(0.6,1.25$, and $6 \mathrm{mM}$ at the low concentration side). It shows a steep decrease in the $\mathrm{pH}$ range 5 to 3 and around $\mathrm{pH}$ 3 it reverses its sign. The scaled membrane potential at a high concentration $(200 \mathrm{mM})$ is close to zero. The lowering of the membrane potential for a lower $\mathrm{pH}$ indicates a reduction in membrane selectivity toward cations. Here, a membrane potential of 0 means non-selective ion passage. This can be explained as arising due to the reduction of surface charge at the membrane pores. At the higher $\mathrm{pH}$ range $(5-10)$ potential surface charge groups are more dissociated or more hydroxide is adsorbed. At this point, the surface charge density remains constant with increasing $\mathrm{pH}$. With a decrease in $\mathrm{pH}$, the surface charge reduces via protonation as the hydroxide concentration is negligible. This neutralizes the effective surface charge and ultimately introduces a positive surface charge, indicated by the sign change of the membrane potential. The variation of surface charge with $\mathrm{pH}$ will be discussed in detail later on. At a high $\mathrm{KCl}$ concentration, the surface charge of the graphene surface is more screened by the counterions. At this point, the potential generated is partly due to the difference in diffusivities of cations and anions inside the membrane. For $\mathrm{KCl}$, this so-called diffusion potential is close to zero. For this reason, the potential at a higher concentration is close to zero and is independent of $\mathrm{pH}$.

Figure 2 shows the variation of the membrane potential with $\mathrm{KCl}$ concentration for different $\mathrm{pH}$ values $(\mathrm{pH} \mathrm{3,} \mathrm{pH} 4$, and

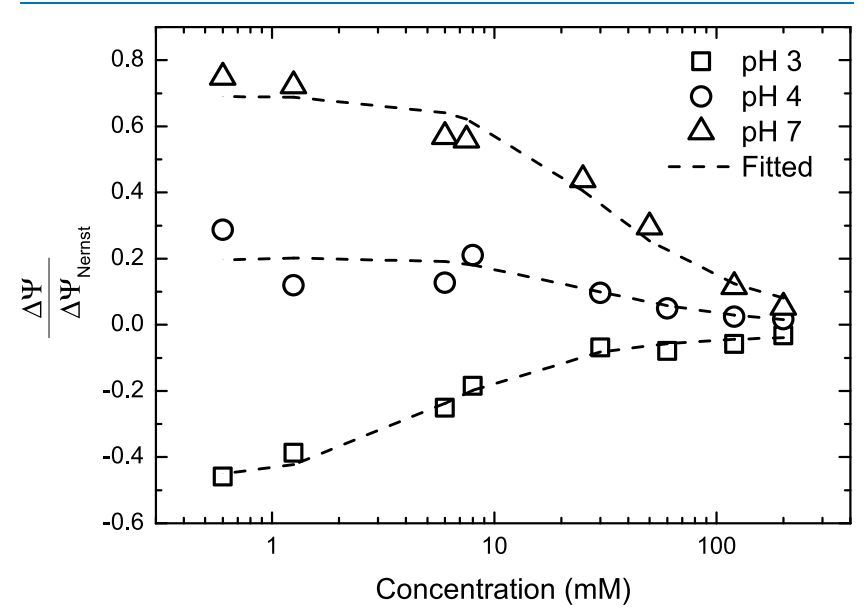

Figure 2. Scaled membrane potential with Nernst potential vs low concentration at $\mathrm{pH} 3, \mathrm{pH} 4$, and $\mathrm{pH} 7$.

$\mathrm{pH}$ 7). For the three $\mathrm{pH}$ values, the potential displays a plateau for low $\mathrm{KCl}$ concentrations $(0.6-8 \mathrm{mM})$. At a high concentration, this value is close to zero. This trend in membrane potential resembles the sigmoid curve observed for dense ion exchange membranes. For $\mathrm{pH} 7$ and $\mathrm{pH} 4$, a positive Donnan plateau is observed, showing a cation selective behavior. In the case of $\mathrm{pH} \mathrm{4,} \mathrm{this} \mathrm{plateau} \mathrm{is} \mathrm{quite} \mathrm{low,}$ indicating a low surface charge compared to $\mathrm{pH}$ 7. For $\mathrm{pH} 3$, the Donnan plateau is negative, which is indicative of anion 
selectivity. The membrane potential at high $\mathrm{KCl}$ concentrations is close to zero for all three $\mathrm{pH}$ values. In this concentration range, we expect the diffusion potential to dominate. The membrane potential vs concentration data is fitted with a modified version of Teorell-Meyer-Sievers (TMS) model as described in our previous paper. ${ }^{14,30-33}$ The best-fit value for fixed ion concentration $\left(\bar{C}_{R}\right)$, the ideality factor $(\alpha)$, and the diffusivity ratio for anion to cation $\left(\frac{\bar{u}_{-}}{\bar{u}_{+}}\right)$are given in Table 1 . Recall that the lower membrane potential

Table 1. Best-Fit Parameters of the Modified TMS Model for the Three $\mathrm{pH}$ Values and Their $95 \%$ Confidence Intervals

\begin{tabular}{cccc}
$\mathrm{pH}$ & $\bar{C}_{R}(\mathrm{mM})$ & $\alpha$ & $\bar{u}_{-} / \bar{u}_{+}$ \\
3 & $-14 \pm 4$ & $0.46 \pm 0.04$ & $1.07 \pm 0.03$ \\
4 & $71 \pm 89$ & $0.20 \pm 0.04$ & $1.00 \pm 0.1$ \\
7 & $78 \pm 28$ & $0.70 \pm 0.04$ & $0.96 \pm 0.1$ \\
\hline
\end{tabular}

compared to the ideal value can be a result from the concentration gradient present inside the PET support pore as well as from graphene membrane non-idealities like defects, large pores or insufficiently high surface potentials. In our previous study, the $\alpha$ factor, which was introduced to the TMS model to take care of various non-idealities, was an empirical one. ${ }^{14}$ Here, we associate a clearer physical meaning to it. We demonstrate that a substantial contribution to this deviation from the Nernst potential can be plausibly attributed to the effect of the PET pores. With a higher $\bar{C}_{R}$, the membrane is expected to have higher rejection of co-ions. At $\mathrm{pH} 7$, the $\bar{C}_{R}$ value is around $78 \mathrm{mM}$, which is high in value compared to that at $\mathrm{pH}$ 3. At $\mathrm{pH} 3$, the surface charge changes its sign. For $\mathrm{pH} 4$, the membrane potential is close to zero and the Donnan plateau is very low compared to the Nernst potential. This indicates a transition region between the Donnan and diffusion plateaus that is difficult to locate, resulting in a $\bar{C}_{R}$ value with a large error (the $95 \%$ confidence interval passes through zero). This implies that $\bar{C}_{R}$ is equivalent to zero in our model, which is consistent with $\mathrm{pH} 4$ being close to the isoelectric point of our graphene pores. It is important to mention that, for our system, $\bar{C}_{R}$ is not a physical parameter but instead a fitting parameter, which provides an indication on the surface charge in our system. It is also interesting to see that the $\alpha$ values are larger for $\mathrm{pH} 3$ and $\mathrm{pH} 7$, at which the graphene membrane is presumably charged. This nicely correlates to the fact that the diffusion resistance of charged nanopores is larger compared to uncharged nanopores, and therefore the fraction of potential drop occurring across the pores is also larger. The general results are highly consistent with our streaming current data, which will be presented in the following section.

To support the variation of surface charge with $\mathrm{pH}$, streaming current measurements have been carried out. As mentioned in Section 2, the zeta potential is calculated from the streaming current value by the Helmholtz-Smoluchowski equation. Figure 3 shows the variation of the zeta potential with varying $\mathrm{pH}$ (11 to 3 ) at a $15 \mathrm{mM} \mathrm{KCl}$ concentration. At higher $\mathrm{pH}$ values (11 to 7 ), the zeta potential values remain negative at an almost constant value. With a decrease in $\mathrm{pH}(7$ to 3 ), the zeta potential becomes less negative and, at low $\mathrm{pH}$ values, becomes positive. These results are consistent with the membrane potential experiments and can be explained by proton dissociation processes.

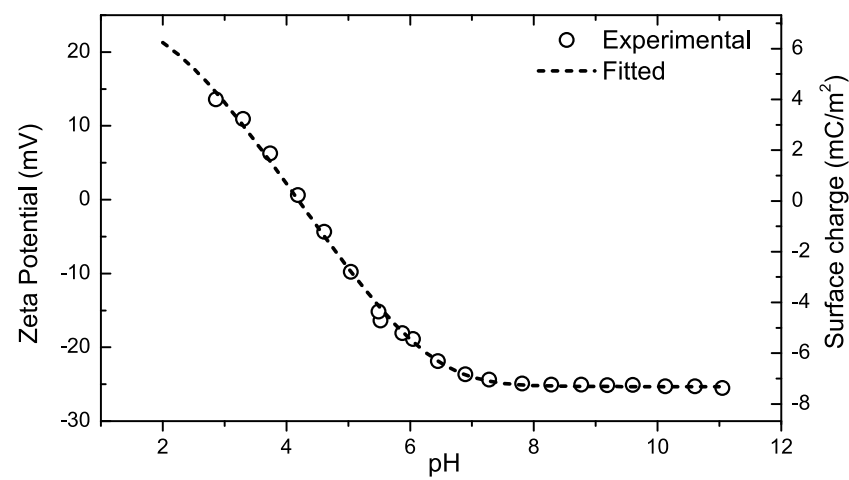

Figure 3. Zeta potential (from streaming potential measurements) fitted with the 1-pK model. The surface charge density derived from the zeta potential is plotted in the right axis.

Using the 1-pK BSM model, the zeta potential of the membrane surface can be used to estimate the membrane surface charge density. The estimated surface charge density is also plotted in Figure 3. In eq 3, the surface potential and surface charge are related nonlinearly. Since the absolute value of zeta potential is less than $25 \mathrm{mV}$, the nonlinearity is relatively weak and therefore both the zeta potential and surface charge have been plotted in two linear scales on the two sides of Figure 3. This result has a good correlation with the fitted $\bar{C}_{R} \cdot \bar{C}_{R}$, multiplied with the sign of the surface charge (-ve in our case) and the Faraday constant, is converted to the charge in $\mathrm{C} / \mathrm{cm}^{3}$ as shown in Table 2 . The table shows that the

Table 2. Surface Charge Density (from Streaming Potential Measurements) and Volume Charge Density (from TMS Fitting) at Varied $\mathrm{pH}$

\begin{tabular}{ccc}
$\mathrm{pH}$ & $\begin{array}{c}\text { surface charge density from streaming } \\
\text { current }\left(\mathrm{mC} / \mathrm{m}^{2}\right)\end{array}$ & $\begin{array}{c}\text { volume charge density } \\
\left(-F \bar{C}_{R}\right)\left(\mathrm{C} / \mathrm{cm}^{3}\right)\end{array}$ \\
3 & 3.88 & 1.32 \\
4 & 0.67 & -6.82 \\
7 & -6.94 & -7.48 \\
\hline
\end{tabular}

$-F \bar{C}_{R}$ values exhibit a similar trend with $\mathrm{pH}$ compared to the surface charge density. The ratio of the surface charge density from streaming current measurements and the volumetric charge density from the TMS fit could theoretically represent a lengthscale characteristic of the thickness of the charged zone. At $\mathrm{pH} \mathrm{7,} \mathrm{where} \mathrm{measured} \mathrm{values} \mathrm{are} \mathrm{most} \mathrm{significant,} \mathrm{this}$ corresponds to a thickness of about $1 \mathrm{~nm}$. It is important to note that the streaming current measurement takes the average over the whole graphene surface and therefore does not account for local charge heterogeneities. This means that the streaming potential measurement largely measures graphene vs a graphene nanopore as the density of pores is approximately 5 $\mu \mathrm{m}^{-2}$. This implies that the surface charge in the streaming current measurement is controlled by the non-porous graphene rather than the nanopores. The transport of ions, however, is determined by the local surface charges at the nanopores with a diameter of $1-10 \mathrm{~nm} .{ }^{14}$

Independent of the mechanism of charge regulation, it is assumed that the trend in estimated zeta potential gives a reasonable indication of the trend in local zeta potential at the pores relevant for ion transport through the pore. Disregarding additional ion adsorption, the zeta potential as a function of $\mathrm{pH}$ can be described quite well by the model as shown in Figure 3. The resulting fitting parameters for a $\mathrm{pK}$ value of 4.2 
are a Helmholtz capacity $C_{1}$ of $14.4 \mu \mathrm{F} / \mathrm{cm}^{2}$ and a surface site density $N_{s}$ of $0.0914 \mathrm{~nm}^{-2}$. The specific integral capacitance of the electric double layer $\left(C_{\mathrm{EDL}}=\sigma_{0} / \Psi_{0}\right)$ was calculated using the model and was around $9.6 \mu \mathrm{F} / \mathrm{cm}^{2}$ within the considered $\mathrm{pH}$ range, ${ }^{34}$ which is in the order of magnitude previously observed for graphene ${ }^{35}\left(2.5 \mu \mathrm{F} / \mathrm{cm}^{2}\right)$ and reduced graphene oxide $^{36}\left(6.5 \mu \mathrm{F} / \mathrm{cm}^{2}\right)$. Hydroxide adsorption was proposed by Bepete et al. ${ }^{22}$ for single-layer graphene sheets dispersed in water, which resulted in a similar zeta potential and point of zero charge as observed in this work. Charge reversal to positive surface charge could be caused by adsorption of protons. For graphene oxide, it is known that carboxylic acid groups are located at the edges. ${ }^{1,37,38}$ This was also observed for reduced graphene oxide. If the nanopores are oxidized during the fabrication process, carboxylic acid groups could likely form. The determined $\mathrm{pK}$ value for these groups present in reduced graphene oxide is around $8 .{ }^{16}$ Carboxylic acid as the sole charge regulation mechanism, however, would not explain the positive zeta potential observed below $\mathrm{pH} 4$.

The presence of the PET support can affect the local concentration near the graphene pore. The support pore volume can in the worst case be considered to be unmixed, so a diffusion-based ion concentration gradient can appear in the PET pore. The challenge of maintaining a concentration difference during stationary membrane potential measurements with composite/asymmetric membranes has been recognized before. Yaroshchuk et al. have experimentally addressed this by the concentration-step technique, considering the transient response of membrane potential. ${ }^{39,40}$ As it is difficult to experimentally access this region in our case, we have analyzed the ion concentration and potential distributions via numerical simulations. For this, we assume a well-mixed bulk as upper and lower boundary conditions (with fixed reservoir concentrations), while the ion concentration and potential distribution are solved via the Poisson and NernstPlanck equations, with an order of magnitude estimate for the Stern layer thickness $(\sim 0.5 \mathrm{~nm}$ for $\mathrm{KCl}){ }^{41,42}$ This allows for estimating the potential difference and concentration distribution expected across such a system. A schematic of the geometry considered is shown in the Supporting Information. In this model, the graphene layer is assumed to be infinitely thin and the model is solved in cylindrical coordinates with a symmetry plane at $r=0$. By assigning the graphene a surface potential, the overall membrane potential between the perfectly mixed reservoirs can be assessed along with the impact of including the unmixed PET support.

The PET pore is unmixed in order to assess the impact of the ion transport in the support on the resulting membrane potential. For this, we kept the surface potential of the PET pore to a zero value. The graphene pore contains a fixed surface potential. The cases with a substrate (PET pore) and without were considered in order to simulate the resulting membrane potential vs concentration. The difference between these values was used to determine the potential impact of the PET support on the deviation from ideality $(\alpha)$ but also possible shifts in the curves. In these simulations, only $\mathrm{KCl}$ was considered for simplicity to start as the goal was to illustrate the possible impact of the support on the resulting membrane potential. The simulations were carried out using the finite element method in the software package COMSOL Multiphysics 5.5 .

The graphene pore radius (or pore size distribution) is estimated between 1 and $10 \mathrm{~nm}$. The surface potentials are limited to a range of -150 to $150 \mathrm{mV}$ approximately based on the typical range of zeta potential being between -100 and 100 $\mathrm{mV} .{ }^{43}$ We found that, when considering effective pore radii (meaning it includes the Stern layer) larger than $2.5 \mathrm{~nm}$, extremely high surface potentials were required in order to obtain simulation values close to the experimentally observed membrane potential values in the Donnan-dominated regime (low absolute concentrations). Even with including activity coefficient effects, such high surface potentials also led to substantial offset in the high concentration (diffusion) regime. As the graphene pore size increases, the impact of the PET pore radius on the observed deviation from ideal behavior also increases.

Figure 4 shows an example case with a graphene surface potential of $-85 \mathrm{mV}$ and an effective pore radius of $1.5 \mathrm{~nm}$

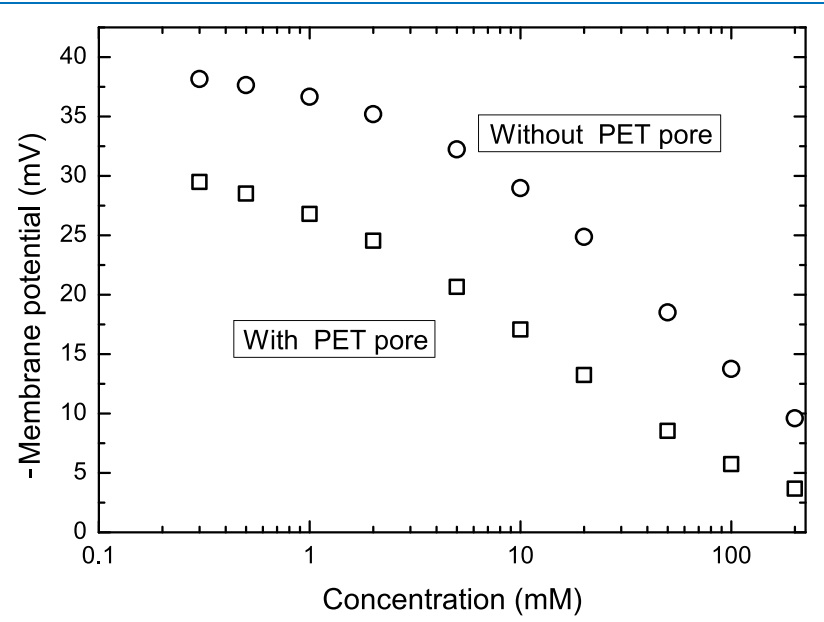

Figure 4. Simulated -membrane potential $(\mathrm{mV})$ vs concentration $(\mathrm{mM})$ with and without PET support. Graphene pore radius $=1.5$ $\mathrm{nm}$; graphene surface potential $=-85 \mathrm{mV}$.

with and without a $13 \mu \mathrm{m}$ long PET pore. On the low concentration reservoir side, the PET pore radius is $250 \mathrm{~nm}$ and, in contact with graphene, the radius is $130 \mathrm{~nm}$ as per SEM images (see the Supporting Information). The results clearly show that a substantial portion of the deviation from ideal Nernst selectivity can be attributed to a concentration gradient within the PET pore. In the case of $-85 \mathrm{mV}$ and $1.5 \mathrm{~nm}$ radii graphene pores, the PET pore effect can explain approximately $75 \%$ of the deviation from the Nernst potential attributed to diffusion within the pore, meaning $75 \%$ of $(1-\alpha)$ can be explained via diffusion through the PET pore. For $2.5 \mathrm{~nm}$ radii graphene pores, a surface potential of $-110 \mathrm{mV}$ was needed to capture the low concentration behavior of the system. In this case, the PET pore can contribute up to $85 \%$ of the deviation from the ideal Nernst potential, meaning $85 \%$ of $(1-\alpha)$ would be explained via diffusion through the PET pore. For the case without the influence of the support, surface potentials of approximately $-60 \mathrm{mV}$ also yielded good agreement with experimental observations.

The PET support is able to explain a reduction in measured membrane potential due to its effect on the local ion concentration distribution. The $\mathrm{pH}$ dependency that we report for the graphene surface charge and ion selectivity is, however, governed by the graphene pore characteristics. 


\section{CONCLUSIONS}

In summary, we have observed the variation of the potential of graphene with $\mathrm{pH}$ during membrane potential measurements. The membrane potential decreases with decreasing $\mathrm{pH}$. At a high $\mathrm{pH}$, the surface charge groups remains negative, resulting in cation selectivity of the membrane, mostly independent of the hydroxyl concentration. With a decrease in $\mathrm{pH}$, the membrane surface charge group becomes more protonated. This is confirmed by both the surface streaming potential measurements as well as the membrane potential measurements. At high salt concentrations, little change in membrane potential with $\mathrm{pH}$ is observed because of the screening of the surface charge groups. For low salt concentrations, the membrane selectivity is directly influenced by the $\mathrm{pH}$ and inverses near a $\mathrm{pH}$ of 4 . The full Nernst potential, indicating ideal selectivity, is never obtained in our measurements. From numerical simulations, we have concluded that the main reason for the deviation from ideal Nernst potential is due to the PET support layer. By fitting our data with the 1-pK BSM model, the surface $\mathrm{pK}$ is determined around 4 , which is quite different from the expected $\mathrm{pK}$ for surface carboxylic acid groups. Both measurements (membrane potential and streaming potential) indicate the same trends regarding surface charge regulation by bulk $\mathrm{pH}$.

\section{ASSOCIATED CONTENT}

\section{(s) Supporting Information}

The Supporting Information is available free of charge at https://pubs.acs.org/doi/10.1021/acsomega.0c03958.

The effect of the PET layer on the membrane potential for the composite membrane (PDF)

\section{AUTHOR INFORMATION}

\section{Corresponding Authors}

Jeffery A. Wood - Soft matter, Fluidics and Interfaces, Faculty of Science and Technology, University of Twente, 7500 AE Enschede, The Netherlands; $\odot$ orcid.org/0000-0002-94381048; Email: j.a.wood@utwente.nl

Rob G. H. Lammertink - Soft matter, Fluidics and Interfaces, Faculty of Science and Technology, University of Twente, 7500 AE Enschede, The Netherlands; (i) orcid.org/00000002-0827-2946; Phone: +31 53 4894798;

Email: r.g.h.lammertink@utwente.nl

\section{Authors}

Mandakranta Ghosh - Soft matter, Fluidics and Interfaces, Faculty of Science and Technology, University of Twente, 7500 AE Enschede, The Netherlands

Moritz A. Junker - Soft matter, Fluidics and Interfaces, Faculty of Science and Technology, University of Twente, 7500 AE Enschede, The Netherlands

Robert T. M. van Lent - Soft matter, Fluidics and Interfaces, Faculty of Science and Technology, University of Twente, 7500 AE Enschede, The Netherlands

Lukas Madauß - Fakultät für Physik und CENIDE, Universität Duisburg-Essen, 47057 Duisburg, Germany; ○ orcid.org/0000-0003-2556-5967

Marika Schleberger - Fakultät für Physik und CENIDE, Universität Duisburg-Essen, 47057 Duisburg, Germany; (1) orcid.org/0000-0002-5785-1186

Henning Lebius - Normandie University, ENSICAEN, UNICAEN, CEA, CNRS, CIMAP, 14032 Caen, France
Abdenacer Benyagoub - Normandie University, ENSICAEN, UNICAEN, CEA, CNRS, CIMAP, 14032 Caen, France

Complete contact information is available at:

https://pubs.acs.org/10.1021/acsomega.0c03958

Notes

The authors declare no competing financial interest.

\section{ACKNOWLEDGMENTS}

The work is performed under the NU-TEGRAM project funded by NWO-I with project number 15FLAG02, by ANR under number ANR-15-GRFL-0002, and DFG under number SCHL 384/16-1, project number 279028710. The authors thank the membrane science and technology cluster for the laboratory facility and all our project partners of NUTEGRAM for useful discussions. We also thank the facility of GANIL, France for ion beam irradiation.

\section{REFERENCES}

(1) O’Hern, S. C.; Boutilier, M. S. H.; Idrobo, J.-C.; Song, Y.; Kong, J.; Laoui, T.; Atieh, M.; Karnik, R. Selective Ionic Transport Through Tunable Subnanometer Pores in Single-layer Graphene Membranes. Nano Lett. 2014, 14, 1234-1241.

(2) Madauß, L.; Schumacher, J.; Ghosh, M.; Ochedowski, O.; Meyer, J.; Lebius, H.; Ban-d'Etat, B.; Toimil-Molares, M. E.; Trautmann, C.; Lammertink, R. G. H.; Ulbricht, M.; Schleberger, M. Fabrication of Nanoporous Graphene/Polymer Composite Membranes. Nanoscale 2017, 9, 10487-10493.

(3) Rollings, R. C.; Kuan, A. T.; Golovchenko, J. A. Ion Selectivity of Graphene Nanopores. Nat. Commun. 2016, 7, 11408.

(4) Celebi, K.; Buchheim, J.; Wyss, R. M.; Droudian, A.; Gasser, P.; Shorubalko, I.; Kye, J.-I.; Lee, C.; Park, H. G. Ultimate Permeation Across Atomically Thin Porous Graphene. Science 2014, 344, 289292.

(5) Surwade, S. P.; Smirnov, S. N.; Vlassiouk, I. V.; Unocic, R. R.; Veith, G. M.; Dai, S.; Mahurin, S. M. Water Desalination Using Nanoporous Single-layer Graphene. Nat. Nanotechnol. 2015, 10, 459464.

(6) Clochard, M.-C.; Melilli, G.; Rizza, G.; Madon, B.; Alves, M.; Wegrowe, J.-E.; Toimil-Molares, M. E.; Christian, M.; Ortolani, L.; Rizzoli, R.; Morandi, V.; Palermo, V.; Bianco, S.; Pirri, F.; Sangermano, M. Large Area Fabrication of Self-standing Nanoporous Graphene-on-PMMA Substrate. Mater. Lett. 2016, 184, 47-51.

(7) Nebogatikova, N. A.; Antonova, I. V.; Erohin, S. V.; Kvashnin, D. G.; Olejniczak, A.; Volodin, V. A.; Skuratov, A. V.; Krasheninnikov, A. V.; Sorokin, P. B.; Chernozatonskii, L. A. Nanostructuring Few-layer Graphene Films with Swift Heavy Ions for Electronic Application: Tuning of Electronic and Transport Properties. Nanoscale 2018, 10, 14499-14509.

(8) Guirguis, A.; Maina, J. W.; Zhang, X.; Henderson, L. C.; Kong, L.; Shon, H.; Dumée, L. F. Applications of Nano-porous Graphene Materials - Critical Review on Performance and Challenges. Mater. Horiz. 2020, 7, 1218-1245.

(9) Merchant, C. A.; Healy, K.; Wanunu, M.; Ray, V.; Peterman, N.; Bartel, J.; Fischbein, M. D.; Venta, K.; Luo, Z.; Johnson, A. T. C.; Drndić, M. DNA Translocation through Graphene Nanopores. Nano Lett. 2010, 10, 2915-2921.

(10) Schneider, G. F.; Kowalczyk, S. W.; Calado, V. E.; Pandraud, G.; Zandbergen, H. W.; Vandersypen, L. M. K.; Dekker, C. DNA Translocation through Graphene Nanopores. Nano Lett. 2010, 10, 3163-3167.

(11) Ivankin, A.; Henley, R. Y.; Larkin, J.; Carson, S.; Toscano, M. L.; Wanunu, M. Label-Free Optical Detection of Biomolecular Translocation through Nanopore Arrays. ACS Nano 2014, 8, 1077410781.

(12) Yoo, E.; Kim, J.; Hosono, E.; Zhou, H.-s.; Kudo, T.; Honma, I. Large Reversible Li Storage of Graphene Nanosheet Families for Use 
in Rechargeable Lithium Ion Batteries. Nano Lett. 2008, 8, 22772282

(13) Cai, X.; Lai, L.; Shen, Z.; Lin, J. Graphene and Graphene-based Composites as Li-ion Battery Electrode Materials and Their Application in Full cells. J. Mater. Chem. A 2017, 5, 15423-15446.

(14) Ghosh, M.; Jorissen, K. F. A.; Wood, J. A.; Lammertink, R. G. H. Ion Transport Through Perforated Graphene. J. Phys. Chem. Lett. 2018, 9, 6339-6344.

(15) Ghosh, M.; Madauß, L.; Schleberger, M.; Lebius, H.; Benyagoub, A.; Wood, J. A.; Lammertink, R. G. H. Understanding Mono- and Bivalent Ion Selectivities of Nanoporous Graphene Using Ionic and Bi-ionic Potentials. Langmuir 2020, 36, 7400-7407.

(16) Konkena, B.; Vasudevan, S. Understanding Aqueous Dispersibility of Graphene Oxide and Reduced Graphene Oxide through $\mathrm{p} K_{\mathrm{a}}$ Measurements. J. Phys. Chem. Lett. 2012, 3, 867-872.

(17) Shih, C.-J.; Lin, S.; Sharma, R.; Strano, M. S.; Blankschtein, D. Understanding the pH-Dependent Behavior of Graphene Oxide Aqueous Solutions: A Comparative Experimental and Molecular Dynamics Simulation Study. Langmuir 2012, 28, 235-241.

(18) Orth, E. S.; Ferreira, J. G. L.; Fonsaca, J. E. S.; Blaskievicz, S. F.; Domingues, S. H.; Dasgupta, A.; Terrones, M.; Zarbin, A. J. G. pK Determination of Graphene-like Materials: Validating Chemical Functionalization. J. Colloid Interface Sci. 2016, 467, 239-244.

(19) Coleman, J. N. Liquid Exfoliation of Defect-free Graphene. Acc. Chem. Res. 2013, 46, 14-22.

(20) Rodríguez-Pérez, L.; Herranz, M. Á.; Martín, N. The Chemistry of Pristine Graphene. Chem. Commun. 2013, 49, 3721-3735.

(21) Backes, C.; Hauke, F.; Hirsch, A. The Potential of Perylene Bisimide Derivatives for The Solubilization of Carbon Nanotubes and Graphene. Adv. Mater. 2011, 23, 2588-2601.

(22) Bepete, G.; Anglaret, E.; Ortolani, L.; Morandi, V.; Huang, K.; Pénicaud, A.; Drummond, C. Surfactant-free Single-layer Graphene in Water. Nat. Chem. 2017, 9, 347.

(23) Zimmermann, R.; Freudenberg, U.; Schweiß, R.; Küttner, D.; Werner, C. Hydroxide and Hydronium Ion Adsorption-A Survey. Curr. Opin. Colloid Interface Sci. 2010, 15, 196-202.

(24) Piasecki, W. $1 \mathrm{pK}$ and $2 \mathrm{pK}$ Protonation Models in the Theoretical Description of Simple Ion Adsorption at the Oxide/ Electrolyte Interface: A Comparative Study of the Predicted and Observed Enthalpic Effects Accompanying Adsorption of Simple Ions. Langmuir 2002, 18, 4809-4818.

(25) de Lint, W. B. S.; Benes, N. E.; Lyklema, J.; Bouwmeester, H. J. M.; van der Linde, A. J.; Wessling, M. Ion Adsorption Parameters Determined from Zeta Potential and Titration Data for a $\gamma$-Alumina Nanofiltration Membrane. Langmuir 2003, 19, 5861-5868.

(26) Piasecki, W.; Rudziński, W.; Charmas, R. 1-pK and 2-pK Protonation Models in the Theoretical Description of Simple Ion Adsorption at the Oxide/Electrolyte Interface: A Comparative Study of the Behavior of the Surface Charge, the Individual Isotherms of Ions, and the Accompanying Electrokinetic Effects. J. Phys. Chem. B 2001, 105, 9755-9771.

(27) Peeters, J. M. M.; Mulder, M. H. V.; Strathmann, H. Streaming Potential Measurements as A Characterization Method for Nanofiltration Membranes. Colloids Surf., A 1999, 150, 247-259.

(28) Ferraris, S.; Cazzola, M.; Peretti, V.; Stella, B.; Spriano, S. Zeta Potential Measurements on Solid Surfaces for in Vitro Biomaterials Testing: Surface Charge, Reactivity Upon Contact With Fluids and Protein Absorption. Front. Bioeng. Biotechnol. 2018, 6, 60.

(29) Riley, J.; Colloid Science; John Wiley \& Sons, Ltd: 2009; Chapter 2, pp. 14-35.

(30) Tanaka, Y. Ion Exchange Membranes : Fundamentals and Applications; Elsevier: Amsterdam, Netherlands, 2015; pp. 59-66.

(31) Sata, T. Ion Exchange Membranes: Preparation, Characterization, Modification and Application; The Royal Society of Chemistry: Cambridge, U.K., 2004; pp 7-16.

(32) Galama, A. H.; Post, J. W.; Hamelers, H. V. M.; Nikonenko, V. V.; Biesheuvel, P. M. On the Origin of the Membrane Potential Arising Across Densely Charged Ion Exchange Membranes: How
Well Does the Teorell-Meyer-Sievers Theory Work? J. Membr. Sci. Res. 2016, 2, 128-140.

(33) Shang, W.-J.; Wang, X.-L.; Yu, Y.-X. Theoretical Calculation on the Membrane Potential of Charged Porous Membranes in 1-1, 1-2, 2-1 and 2-2 Electrolyte Solutions. J. Membr. Sci. 2006, 285, 362-375.

(34) Oldham, K. B. A Gouy-Chapman-Stern Model of the Double Layer at A (Metal)/(Ionic liquid) interface. J. Electroanal. Chem. 2008, 613, 131-138.

(35) Ji, H.; Zhao, X.; Qiao, Z.; Jung, J.; Zhu, Y.; Lu, Y.; Zhang, L. L.; MacDonald, A. H.; Ruoff, R. S. Capacitance of Carbon-based Electrical Double-layer Capacitors. Nat. Commun. 2014, 5, 3317.

(36) Xu, K.; Ji, X.; Chen, C.; Wan, H.; Miao, L.; Jiang, J. Electrochemical Double Layer Near Polar Reduced Graphene Oxide Electrode: Insights from Molecular Dynamic Study. Electrochim. Acta 2015, 166, 142-149.

(37) Whitby, R. L.; Gun'ko, V. M.; Korobeinyk, A.; Busquets, R.; Cundy, A. B.; László, K.; Skubiszewska-Zięba, J.; Leboda, R.; Tombácz, E.; Toth, I. Y.; et al. Driving Forces of Conformational Changes in Single-layer Graphene Oxide. ACS Nano 2012, 6, 39673973.

(38) Park, S.; Ruoff, R. S. Chemical Methods for The Production of Graphenes. Nat. Nanotechnol. 2009, 4, 217.

(39) Yaroshchuk, A. E.; Makovetskiy, A. L.; Boiko, Y. P.; Galinker, E. W. Non-steady-state Membrane Potential: Theory and Measurements by a Novel Technique to Determine the Ion Transport Numbers in Active Layers of Nanofiltration Membranes. J. Membr. Sci. 2000, 172, 203-221.

(40) Yaroshchuk, A.; Boiko, Y.; Makovetskiy, A. Electrochemical Perm-selectivity of Active Layers and Diffusion Permeability of Supports of An Asymmetric And A Composite NF Membrane Studied by Concentration-step Method. Desalination 2009, 245, 374387.

(41) Moran, J. L.; Posner, J. D. Electrokinetic Locomotion Due to Reaction-induced Charge Auto-electrophoresis. J. Fluid Mech. 2011, 680, 31-66.

(42) Bazant, M. Z.; Chu, K. T.; Bayly, B. J. Current-Voltage Relations for Electrochemical Thin Films. SIAM J. Appl. Math. 2005, $65,1463-1484$.

(43) Kirby, B. J.; Hasselbrink, E. F., Jr. Zeta Potential of Microfluidic Substrates: 1. Theory, Experimental Techniques, And Effects on Separations. Electrophoresis 2004, 25, 187-202. 